\title{
Who Is the Patient at Risk of CMV Recurrence: A Review of the Current Scientific Evidence with a Focus on Hematopoietic Cell Transplantation
}

Jan Styczynski

Received: August 31, 2017 / Published online: December 4, 2017

(C) The Author(s) 2017. This article is an open access publication

\begin{abstract}
Cytomegalovirus (CMV) is an agent of global infection, and its acquisition in a population is characterized by an age-dependent rise in seropositivity. After primary infection, CMV remains in the host cells in latent form, and it can reactivate in the case of immune suppression. The risk of CMV recurrence is dependent on the level of incompetency of the immune system, manifested as an impairment of T-cell immunity, including the presence and function of CMV-specific cytotoxic T lymphocytes. This article presents data on the incidence of $\mathrm{CMV}$ recurrence in groups of immunocompromised patients, including allogeneic hematopoietic stem cell transplantation (HSCT) patients and other groups of patients, based on a summary of reported data. The median rate of CMV recurrence in HSCT recipients was estimated as $37 \%$ after allogeneic transplant and $12 \%$ after autologous transplant, $5 \%$ in patients with nontransplant hematological malignancies, $14 \%$ in recipients
\end{abstract}

Enhanced content To view enhanced content for this article go to http://www.medengine.com/Redeem/ 76DCF0601945D87B.

J. Styczynski ( $\square)$

Department of Pediatric Hematology and Oncology,

Collegium Medicum, Nicolaus Copernicus

University in Toruń, Bydgoszcz, Poland

e-mail: jstyczynski@cm.umk.pl of anti-CD52 therapy, $30 \%$ in solid organ transplant recipients, $21 \%$ in patients with primary immunodeficiencies, $20 \%$ during active replication in HIV-positive patients and $3.3 \%$ during antiretroviral therapy, $7 \%$ in patients with chronic kidney disease, $0.6 \%$ in patients with congenital infection, and $0.6 \%$ in neonates with primary infection. The highest risk of CMV recurrence and CMV disease is reported for HSCT CMV-seropositive recipients, regardless of donor serostatus. The odds ratio (OR) for CMV recurrence is higher for recipient-positive versus recipient-negative CMV serostatus transplants (OR 8.0), donornegative/recipient-positive versus donor-positive/recipient-positive CMV serostatus transplants (OR 1.2), unrelated/mismatched versus matched-family donor transplants (OR 1.6), and acute graft-versus-host-disease versus other diseases (OR 3.2). Other risk factors have minor significance.

Keywords: Cytomegalovirus; Cytomegalovirus disease; Cytomegalovirus recurrence; Hematopoietic stem cell transplantation; Population; Risk factors; Risk group

\section{CYTOMEGALOVIRUS}

Cytomegalovirus (CMV), human herpesvirus 5, is an agent of a global infection, although 
differences in the seroprevalence exist between countries $[1,2]$. CMV acquisition in a population is characterized by an age-dependent rise in seropositivity, and correlates most closely with socioeconomic level and race [2], similarly to other herpesviruses [3-5]. CMV seroprevalence ranges from about $40-50 \%$ in highly developed countries to almost $95 \%$ in developing countries [1], and from about 30\% in childhood to $50 \%$ in women of childbearing age and up to $60-70 \%$ in adults $[2,6]$. On the other hand, the incidence slowly decreases with the calendar year [2]. In populations of industrialized countries, CMV acquisition occurs at a rate of $1-7 \%$ per year $[7,8]$.

After primary infection, CMV always progresses to life-long latency, which is typical for herperviruses. Usually no clinical signs and symptoms of CMV infection occur in the general population, except for possible episodes of fever, hepatitis, or mononucleosis-like syndrome. In the case of immune suppression, latent infection may reactivate, causing CMV infection leading to CMV disease. Thus, CMV remains a major cause of morbidity and death in immunocompromised patients.

In immunocompetent individuals the latent CMV infection is under the control of the immune system. Cellular immunity driven by T cells is responsible for controlling CMV replication [9]; however lack or delayed recovery of CMV-specific $\mathrm{CD}^{+}$and $\mathrm{CD}^{+}$lymphocytes predisposes to CMV recurrence and CMV disease $[10,11]$.

\section{OBJECTIVES AND METHODS}

This article aims to analyze the incidence of CMV recurrence and CMV disease in selected populations of immunocompromised patients. Special attention is given to patients after allogeneic hematopoietic stem cell transplantation (HSCT). For this group of patients, the risk factors for CMV recurrence and disease were analyzed.

To determine the risk of CMV recurrence or CMV disease in selected risk groups, reported data were obtained from published studies registered in PubMed from 1995 to August 2017.
To analyze risk factors in hematopoietic stem cell transplant patients, the search was done with use of the terms "CMV", "risk factor," "allogeneic hematopoietic stem cell transplantation," and "multivariate analysis." All studies analyzing risk factors in multivariate analyses with the numbers of patients at risk, available for summary of the data, were included. Eightyseven possible publications related to CMV infection in hematopoietic stem cell transplant patients were found. After exclusion of nonrelevant, non-English, and review articles, potentially relevant articles were selected. Sufficient data available for further analysis of the role of risk factors were found in nine articles for early or late CMV infection and three articles for CMV disease. In the analysis of the raw data, odds ratios (ORs) and 95\% confidence intervals (CIs) were determined.

As with all retrospective studies based on data mining from published work, data have to be seen with caution because techniques, detection sensitivities, and medical standards in general may differ between different medical centers and different years of publication and cannot simply be compiled.

\section{Compliance with Ethics Guidelines}

This article is based on previously conducted studies and does not involve any new studies of human or animal subjects performed by the author.

\section{DEFINITIONS}

Recently, updated definitions on CMV infection and CMV disease were prepared by the CMV Drug Development Forum [12]. Briefly:

- CMV infection is defined as virus isolation or detection of viral proteins (antigens) or nucleic acid in any body fluid or tissue specimen.

- "CMV replication" indicates evidence of viral multiplication and is sometimes used instead of "CMV infection."

- Primary CMV infection is defined as the first detection of CMV infection in an individual who has no evidence of CMV exposure. 
- Recurrent CMV infection is defined as new CMV infection in a patient with previous evidence of CMV infection in whom the virus has not been detected for at least 4 weeks during active surveillance. Recurrent infection may result from reactivation of latent virus (endogenous) or reinfection (exogenous).

- CMV reinfection is defined as detection of a CMV strain that is distinct from the strain that caused the initial infection.

- CMV reactivation is likely if the two viral strains (prior and current strain) are found to be indistinguishable either by sequencing of specific regions of the viral genome or by use of a variety of molecular techniques that examine genes known to be polymorphic.

- Symptomatic CMV infection is diagnosed in patients developing symptoms (fever with or without bone marrow suppression) and who have CMV virions, antigens, or nucleic acid detectable but with no sign of CMV endorgan disease [3].

- CMV disease is diagnosed in a patient with symptoms and/or signs from the affected organ together with detection of CMV by a test with appropriate sensitivity and specificity from an organ in a biopsy sample or samples from other invasive procedures, with exception for CMV retinitis, for which typical findings from ophthalmologic examination are sufficient [3].

- Definitions on treatment of CMV: "prophylaxis" means that antiviral agents are given to a patient to prevent a primary, reactivated, or recurrent CMV infection; "preemptive therapy" (sometimes called "preemptive prophylaxis") means antiviral agents are given for an asymptomatic CMV infection detected by a screening assay [3].

Current understanding of CMV infection underlines that co-infection with different strains occurs quite frequently, but because of stochasticity of the reactivation event, a single strain may reactivate to give rise to recurrent infection and recrudescent disease $[13,14]$. The problem in clinical studies is that the strain from a latent infection is rarely typed; however CMV strain differences might have significant potential to influence the risk of reactivation/ recurrence. Such strain differences plus the differences in latent viral load depending on the history of primary infection may explain the overall broad ranges of incidences reported in the clinical studies summarized in this review.

\section{WHO IS THE PATIENT AT RISK OF CMV RECURRENCE}

After primary, usually asymptomatic, infection, CMV remains in the host cells in latent form. During immune suppression, especially within the T-cell population, the virus can reactivate and lead to symptomatic CMV infection and CMV end-organ disease. The risk of CMV recurrence is dependent on the level of incompetency of the immune system, manifested as an impairment of T-cell immunity, including the presence and function of CMVspecific cytotoxic T lymphocytes (CTLs). The following groups of immunocompromised individuals are at possible risk of CMV infection (Fig. 1):

- Individuals with primary immunodeficiencies (PIDs);

- Individuals with secondary immunodeficiencies caused by disease of the immune

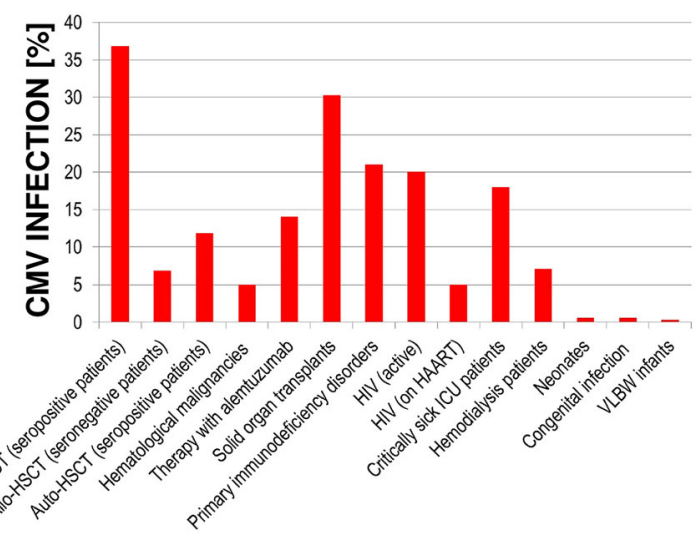

Fig. 1 Groups potentially at risk of CMV infection and the rate of $\mathrm{CMV}$ infection. allo-HSCT allogeneic hematopoietic stem cell transplantation, auto-HSCT autologous hematopoietic stem cell transplantation, HAART highly active antiretroviral therapy, ICU intensive care unit, VLBW very low birth weight 
system: leukemia and other hematological malignancies, human immunodeficiency virus (HIV) infection;

- Recipients of immunosuppressive therapy: HSCT, solid organ transplantation;

- Recipients of drugs suppressing the immune system: anti-CD52, anti-CD20, anti-CD25, anti-tumor necrosis factor (TNF)

- Hemodialysis patients;

- Neonates because of immaturity of the immune system.

\section{Allogeneic Hematopoietic Stem Cell Transplantation Patients}

On the basis of data from large studies, the rate of CMV recurrence after allogeneic HSCT in seropositive patients is $30-80 \%$ [15-21], with a median value of $37 \%$ (Fig. 1). The high rate of $\mathrm{CMV}$ recurrence in this population is caused by extreme immune suppression, which is probably higher than in any other group of patients. Over the years, the rate of CMV disease has decreased significantly in HSCT patients. The rate of CMV recurrence after allogeneic HSCT in seronegative patients is $0-12 \%$ [15-21], with a median value of $7 \%$.

\section{Autologous Hematopoietic Stem Cell Transplant Patients}

The rate of CMV infection after autologous HSCT is significantly lower in comparison with that in CMV-seropositive patients after allogeneic HSCT: it is $0-33 \%$, with a median value of $12 \%$ and a cumulative incidence of $11.83 \%$ (566 of 4784 patients) $[15,22-28]$. This risk can be increased when alemtuzumab or fludarabine is used in seropositive patients.

\section{Hematological Malignancies in Patients Receiving Nontransplant Treatment}

Overall, the rate of CMV infection or recurrence ranges between $2 \%$ and $67 \%$ in nontransplant settings, with a median value of $5 \%$ and a cumulative incidence $4.97 \%$ (340 of 6841 patients) among reported patients $[15,22,25,26,29]$. In pediatric patients with acute lymphoblastic leukemia, CMV recurrence was diagnosed during maintenance therapy in $10 \%$ of children [29]. A higher incidence of CMV antigenemia was observed in lymphoid tumors $(13.6 \%)$ than in myeloid tumors (3.9\%) [22]. The reported risk factors for CMV recurrence in a nontransplant setting included advanced disease, poor performance status, and the use of high-dose steroids, fludarabine, alemtuzumab (anti-CD52 monoclonal antibody), bortezomib (proteasome inhibitor), and rituximab (anti-CD20 monoclonal antibody) [25].

\section{Immunosuppressive Therapy with Alemtuzumab (Anti-CD52 Therapy) or Fludarabine}

The introduction of targeted therapy and monoclonal antibodies in last 20 years, initially in hematology and immunology and then in other medical disciplines, has shown a strong immunosuppressive effect caused by these agents, resulting in an increased risk of infectious complications, including recurrence of herpesviruses. Fludarabine, used both in conditioning before allogeneic HSCT and in nontransplant chemotherapy, has similar potential. According to published data, CMV recurrence developed in $9-67 \%$ of patients who received alemtuzumab, with a median value of $14 \%$ $[25,30,31]$.

\section{Solid Organ Transplants}

CMV infection after solid organ transplantation can occur in both the early and the late posttransplant phase. The incidence of CMV infection and CMV disease depends on various factors, such as the serostatus of the donor and recipient, the type of organ transplant, and the prevention strategies that were used. The rate of CMV infection is $16-56 \%$, with a median value of $30 \%$ [32-36]. The incidence is highest in lung and small bowel transplant recipients and lower in kidney and liver transplant recipients [32]; however, most data are available from the kidney transplant setting. The incidence is associated mainly with the amount of lymphoid 
tissue transplanted with the organ from the donor and the intensity of the immunosuppression. It was shown that CMV viremia influenced all-cause mortality in kidney transplant recipients in multivariate analysis. The following factors increased the risk of CMV viremia: donor-postive $(\mathrm{D}+) /$ recipient-negative $(\mathrm{R}-)$ status; receiving a graft from a deceased donor; and receiving a graft from a donor aged 60 years or older [35].

\section{Primary Immunodeficiencies}

PIDs are disorders of abnormal function or lack of an immune system or part of it. These disorders include combined T- or B-cell immunodeficiencies, predominantly antibody deficiencies, diseases of immune dysregulation, congenital defects of phagocyte number, function, or both, defects in innate immunity, autoinflammatory disorders, complement deficiencies, and other well-defined immunodeficiency syndromes. Because of their nature, these diseases occur almost exclusively in children. For many of them HSCT is the only effective treatment. The number of reports on CMV infection in PID patients is low. Existing rare data suggest the rate of CMV infection is within the range of $9-50 \%$, with a median value of $21 \%$ [37-39]. CMV infection was shown to be a risk factor for death in patients with PID [38, 39].

\section{HIV-Positive Patients}

Patients infected with HIV during the active phase of viral replication had a $5.2-51 \%$ risk of CMV infection, with a median value of $20 \%$ [40-45]. The risk factor was progressive loss of immune function with a CD4 lymphocyte count of less than $100 / \mu \mathrm{L}$. High copy number and subclinical CMV viremia were independent risk factors for death among male HIV-infected adults in South Africa with early HIV disease [43]. Recent data from large cross-sectional studies show a prevalence of $3.3 \%$ for acute CMV infection [46], whereas the current prevalence of CMV viremia in patients infected with HIV starting antiretroviral therapy in developing countries is below $4.5 \%$, and is even lower in developed countries: the median rate of $\mathrm{CMV}$ recurrence is $5 \%$, with a range of $3-36.6 \%$ [40, 44-48].

\section{Patients Receiving Hemodialysis}

CMV recurrence was found in $0-15 \%$ of hemodialysis patients, with a median value of 7\% [49-51], and the CMV seroprevalence reached $92.3 \%$ [50].

\section{Neonates}

Recent reports show the rate of primary CMV infection in neonates to be $0.3-2 \%$, with a median value of $0.6 \%[1,52,53]$, and $0.3 \%$ in very low birth weight infants [54]. The rate of congenital CMV infection is $0.49-0.7 \%$, with a median value of $0.6 \%$; however, it is higher in developing countries, ranging between $1 \%$ and $5 \%[1,55-57]$. Approximately $10 \%$ of patients with congenital CMV infection exhibit symptoms of CMV disease [58].

\section{PHASES AFTER HSCT}

HSCT is a medical procedure of infusion of a product that contains hematopoietic cells, usually derived from bone marrow, peripheral blood, or umbilical cord blood. HSCT is performed to repopulate recipient bone marrow after myeloablative therapy for underlying malignant or nonmalignant disease. HSCT may be autologous or allogeneic. HSCT is connected with severe immune suppression due to initial therapy for primary disease (e.g., chemotherapy), pretransplant conditioning (high-dose chemotherapy and/or total body irradiation, TBI), and immunosuppressive therapy after transplantation. With respect to the risk of infection, immune suppression and immune recovery, three basic posttransplant phases are defined: early phase (from day 0 to day 30); intermediate phase (from day 31 to day 100); and late phase (from day 101, usually up to the end of first year after HSCT) (Fig. 2). 


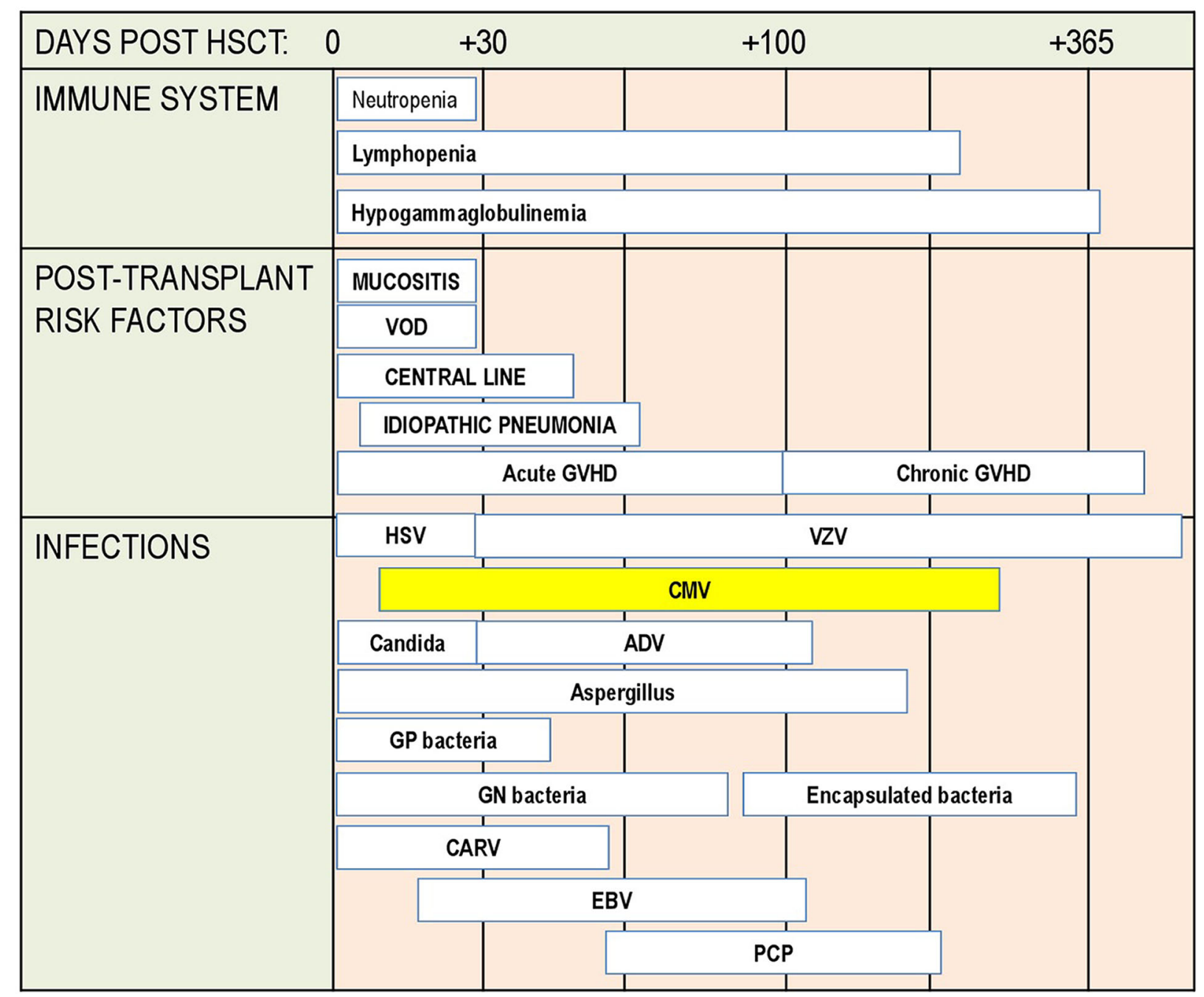

Fig. 2 Posttransplant phases with risk factors and infections in the allogeneic hematopoietic stem cell transplantation (HSCT) setting. ADV adenovirus, CARV community-acquired respiratory viruses, $\mathrm{CMV}, \mathrm{EBV}$

In the early phase, after conditioning therapy in the preengraftment phase, patients undergo an aplastic phase with severe neutropenia until neutrophil levels recover. The infections encountered during the preengraftment phase include primarily bacterial and fungal infections that are reasonably well controlled by medications given for prophylaxis and treatment [59].

The intermediate phase is characterized by cellular immunodeficiency due to a reduced
Epstein-Barr virus, GN gram-negative, GP gram-positive, GVHD graft-versus-host disease, HSV herpes simplex virus, PCP pneumocystis pneumonia, VOD veno-occlusive disease, VZV varicella-zoster virus

number of natural killer cells of the innate immune system and $\mathrm{T}$ cells of the adaptive immune system. Additionally, in the allogeneic hematopoietic stem cell transplantation setting, the risk of acute graft-versus-host-disease (GVHD) and its prophylaxis with immunosuppressive drugs might negatively influence the immune suppression. This renders patients especially susceptible to viral recurrences, including recurrences of CMV and Epstein-Barr virus. 
The late phase is usually characterized by slow reconstitution of $\mathrm{B}$ and $\mathrm{T}$ lymphocytes and prolonged hypogammaglobulinemia. The possible presence of chronic GVHD and respective therapy may severely impair the immune status of an allogeneic hematopoietic stem cell transplantation recipient.

HSCT is nowadays regarded as the most invasive intervention into the patient's immune system. Transplantation of hematopoietic cells leads to full repopulation of bone marrow with hematopoietic cells originating from the donor. It results in exchange of hematopoietic cells, cells in bone marrow and peripheral blood, including white blood cells, red blood cells, and platelets. Consequently, it also results in full exchange of the immune system, which is called "100\% donor chimerism."

Both latent (herpesviruses) and endemic (community-acquired respiratory viruses) viral infections are frequent in patients after HSCT. Recurrence of the latent infection is influenced by immunosuppression, the type of the graft, the serostatus of the donor and recipient, preemptive therapy, antiviral prophylaxis, immunosuppressive prophylaxis or treatment, and the presence of GVHD [60]. Recipient cells infected with CMV remain in the body and CMV can reactivate after HSCT, or can be transferred from the donor. An immunologically incompetent person is not protected by CMV-specific $\mathrm{CD}^{+}$and $\mathrm{CD}^{+}{ }^{+}$cells. Additionally, the intensity of immunosuppression contributes to the lack of cellular response against CMV [60].

CMV infection is a serious infection for the immune system and can adversely affect transplant outcomes, increasing organ toxicity directly via CMV infection itself and indirectly via associated side effects of antiviral therapy. This leads to an increased risk of bacterial and fungal infections. The most frequent clinical manifestations of CMV disease in immunosuppressed patients are pneumonia, hepatitis, bone marrow suppression, retinitis, and enteritis. CMV infection decreases survival after HSCT $[16,61]$.

\section{TIME TO CMV RECURRENCE AND CMV DISEASE}

CMV can reactivate after HSCT usually during the early phase, and reactivation was reported even at day 1 after HSCT. The range of possible times to recurrence is very wide, up to day 918. Recurrence is dependent mainly on prolonged immunosuppressive therapy for chronic GVHD. Nevertheless, most CMV recurrences occur between 2 and 4 month after HSCT, with a median time of day 44 [17, 21, 62-64]. Since antiviral prophylaxis may delay the reconstitution of CMV-specific T-cell lymphocytes, this may increase the risk of development of late CMV disease, occurring after day 100 [64, 65]. The median time to development of CMV disease was 104 days (range 39-200 days) [21].

Obviously, CMV disease develops after CMV recurrence (Fig. 3). Most episodes of CMV disease occur after CMV prophylaxis has been completed or a typical schedule of screening for CMV recurrence and preemptive therapy has finished. They often happen after day 100 after HSCT. Surprisingly, in spite of the continuous risk of CMV recurrence in patients receiving immunosuppressive therapy for chronic GVHD after day 200, the risk of CMV disease is lower because of regular screening for CMV recurrence, and the use of preemptive therapy.

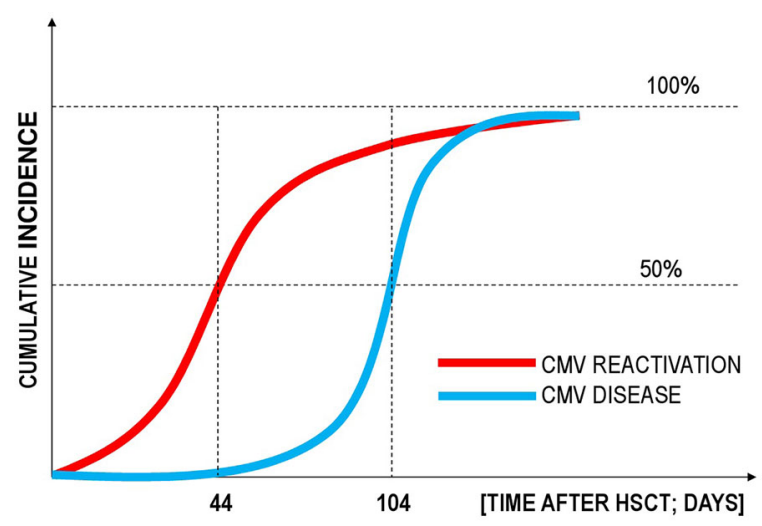

Fig. 3 Estimated time-dependent cumulative incidence of CMV reactivation and CMV infection after allogeneic hematopoietic stem cell transplantation (HSCT) 


\section{RISK FACTORS FOR CMV RECURRENCE IN ALLOGENEIC HEMATOPOIETIC STEM CELL TRANSPLANTATION PATIENTS}

\section{Transplant-Related Background}

The risk factors for CMV recurrence after allogeneic HSCT depend on the recipient (CMV serostatus; age), donor [CMV serostatus match; age; sex match; type of family/unrelated donor (UD); HLA match; stem cell source], transplant (intensity of myeloablative or reduced-intensity conditioning; type of conditioning-TBI based or chemotherapy based; T-cell depletion), immunosuppressive treatment (prophylaxis, occurrence and treatment for acute and/or chronic GVHD; specific immunosuppressive drugs used in prophylaxis and therapy), and immune recovery after HSCT (speed of immune reconstitution for natural killer cells, B cells, $\mathrm{T}$ cells; recovery of CMV-specific CTLs) (Fig. 4).

Some reports indicate the role of other factors such as the experience of the center and improvement in all kinds of antimicrobial prophylaxis (e.g., regarding blood products, infections with other herpesviruses, the use of preemptive prophylaxis, or race of the patient). In analysis of studies by multivariate analysis for risk factors for $\mathrm{CMV}$ recurrence in patients after allogeneic HSCT, donor-negative (D-)/recipient-positive $(\mathrm{R}+)$ pretransplant $\mathrm{CMV}$ serostatus had a major role as a risk factor

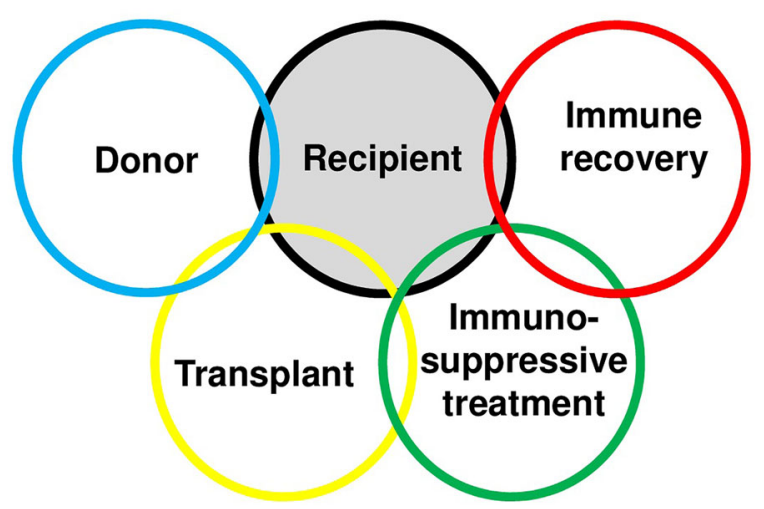

Fig. 4 Risk factors for CMV reactivation in allogeneic hematopoietic stem cell transplantation patients
[18, 21, 62, 63, 66-68]. Most of these studies indicated also other risk factors: UD or mismatched donor (MMD) transplant [67-69], acute GVHD [18, 21, 62, 63, 66-68], and older age $[18,21,62,63,66-69]$. Age should be regarded as a continuous variable, since various cutoff values were given: 18, 41, and 50 years. This characteristic clearly corresponds to an age-dependent rate of CMV seropositivity in the general population [2]. Several other risk factors were found in these analyses: bone marrow as a stem cell source [67], reduced-intensity conditioning [18], TBI-based conditioning [68], year of transplant before 2004 [18], herpes simplex virus infection [63], non-Caucasian race [18], no T-cell depletion [66], and use of steroids [68]. A protective effect of sirolimus use in GVHD prophylaxis was shown in one study [67]. Sirolimus possibly has a protective effect against CMV infection because of the inhibition of cellular signaling pathways that are triggered during CMV infection for the synthesis of viral proteins. It has also antiproliferative properties, and thus probably inhibits the kinetics of CMV replication [70, 71].

With regard to risk factors for both CMV recurrence and CMV disease, the load of latent viral DNA established during primary infection should be taken into account. It is obvious that the risk of recurrence starting from single latent viral genomes critically depends on the number of latent viral genomes present in host tissues, as recurrence is a stochastic event with a probability that increases with increasing number of genomes. Admittedly, this is difficult to document in clinical studies, but one can learn from animal models that within seropositive individuals the latent viral DNA load depends on the age-dependent (immune maturity-dependent) extent of virus replication during primary infection and determines the risk of recurrence during immunosuppression, rather than seropositivity as such, which just indicates a preceding infection. Specifically, infection in neonates or early childhood compared with infection in adults leads to seropositivity, but latent viral load and risk of recurrence are much higher when primary infection occurs early in life [72]. 


\section{The Role of Donor and Recipient CMV Serostatus}

There are two possibilities for donor CMV status including anti-CMV immune response:

1. The donor is CMV naïve, not infected with $\mathrm{CMV}$, and without anti-CMV-IgG (CMV seronegativity). As a result there are no CMV-specific CTLs, among both the $\mathrm{CD}^{+}$and the $\mathrm{CD}^{+}$population. There is no risk of transfer of CMV infection to the transplant recipient, but also there is no transfer of CMV-specific CTLs to the recipient. This is of benefit for a CMV-naïve recipient but not for a CMV-seropositive recipient.

2. The donor is CMV IgG seropositive. This results from CMV primary infection and the latent phase of the virus in the immune system of the donor, with the presence of CMV-specific CTLs. In this case, transplantation of hematopoietic stem cells and transfer of the immune system to the recipient leads to transfer of both $\mathrm{CMV}$ in the latent phase and CMV-specific CTLs. Obviously, the donor's immune system having CMV-specific CD4 and CD8 cells protects against recurrence of CMV. This might be partially beneficial for the recipient infected with CMV because of the transfer of CMV-specific CTLs. The benefit is very limited because the number of CMVspecific CTLs transferred is low. Additionally, the recipient is being treated with immunosuppressive agents to prevent GVHD. On the other hand, in the case of a CMV-seronegative recipient, there is the risk of CMV infection being transferred from the donor, since CMV infection can be transmitted from the donor with hematopoietic stem cells. This might happen even though CMV-specific CTLs are also transferred.

The CMV serostatus of the donor and recipient before transplantation significantly influences the incidence of CMV recurrence (Table 1). The immunosuppression status of the recipient is the most important factor for CMV infection. Seropositive recipients constitute the group with the highest risk of CMV recurrence,
Table 1 Risk of recurrence of CMV with respect to donor/recipient serostatus in the allogeneic hematopoietic stem cell transplantation setting

\begin{tabular}{llc}
\hline $\begin{array}{l}\text { Recipient CMV } \\
\text { serostatus }\end{array}$ & \multicolumn{2}{l}{ Donor CMV serostatus } \\
\cline { 2 - 3 } & Negative & Positive \\
\hline Negative & $3.1 \%(143 /$ & $12.9 \%(322 /$ \\
& $4681)$ & $2503)$ \\
Positive & $35.8 \%(1829 /$ & $32.1 \%(2719 /$ \\
& $5113)$ & $8461)$ \\
\hline
\end{tabular}

Raw data from $[16,17,63,66,67,73]$

whereas the lowest risk of CMV recurrence after HSCT is in the group of patients with $\mathrm{D}-/ \mathrm{R}-$ serotype.

In several studies the differences between $\mathrm{D}+/ \mathrm{R}+$ and $\mathrm{D}-/ \mathrm{R}+$ serostatus were analyzed. The incidence of CMV recurrence in $\mathrm{D}-/$ $\mathrm{R}+$ transplant recipients was significantly higher than that in $\mathrm{D}+/ \mathrm{R}+$ transplant recipients, with ORs ranging from 1.8 to 6.6 $[60,62,64]$. From the data presented in Table 1 , the overall risk of CMV recurrence is higher in $\mathrm{D}-/ \mathrm{R}+$ transplant recipients than in $\mathrm{D}+/$ $\mathrm{R}+$ transplant recipients (OR 1.18, 95\% CI $1.09-1.26, p<0.0001)$.

The benefit of serological match $(\mathrm{D}+/$ $\mathrm{R}+$ ) over $\mathrm{D}-/ \mathrm{R}+$ results from the transfer with the graft of CMV-specific CTLs and antiviral cytokines from a seropositive donor. The memory cells can possibly help to develop an immune response against the virus. In case of $\mathrm{D}-/ \mathrm{R}+$ transplants, immunological anti-CMV reconstitution is prolonged because of the lack of CMV-specific memory $\mathrm{T}$ cells, so the risk of CMV infection is higher.

CMV-seropositive donors usually produce CMV-specific CTLs. This results in a durable CMV-specific CTL response after HSCT and better protection from CMV disease [60]. In contrast, the number of CMV-specific CTLs in $\mathrm{D}-/ \mathrm{R}+$ transplant recipients is very low, so antiCMV-specific T-cell recovery is delayed or absent during the first month after HSCT and the risk of CMV infection is higher in $\mathrm{D}-/$ $\mathrm{R}+$ transplant recipients compared with $\mathrm{D}+/ \mathrm{R}+$ or $\mathrm{D}+/ \mathrm{R}-$ transplant recipients [60]. 
In comparison with CMV-seronegative donors (D-), grafts from CMV-seropositive donors (D+) contain more antiviral cytokines: TNF- $\alpha$, interferon- $\gamma$, and chemokine macrophage inflammatory protein $1 \beta$, as well as the degranulation marker CD107 [62].

\section{Role of Donor Type and GVHD}

Overall, data on CMV recurrence from 23,791 allogeneic hematopoietic stem cell transplantations are available [16-18, 63, 66, 67, 73], mainly in leukemias; among them the CMVseropositive rate of recipients was $63.7 \%$ $(15,145 / 23,791)$. From the published data, recurrence occurred in $36.8 \%$ of CMV-seropositive recipients $(5572 / 15,145)$ and in $6.8 \%$ of CMV-seronegative recipients $(584 / 8646)$. The CMV-seropositive recipients had eightfold higher odds of CMV recurrence than the CMVseronegative recipients (OR 8.04, 95\% CI 7.34-8.74, $p<0.0001$ ).

With respect to the type of donor, recurrence occurred in $44.6 \%$ of UD/MMD transplant recipients $(1077 / 2416)$ and in $33.8 \%$ of matched-family donor (MFD) transplant recipients $(879 / 2601)[63,66,67,73]$. The risk of CMV recurrence was higher in UD/MMD transplants than in MFD transplants (OR 1.58, 95\% CI $1.40-1.77, p<0.0001)$.

CMV recurrence occurred in $60.1 \%$ of patients $(885 / 1472)$ with acute GVHD and in $32.1 \%$ of patients $(892 / 2780)$ without acute GVHD [63, 67, 73]. The risk of CMV recurrence was higher in acute GVHD patients than in patients without acute GVHD (OR 3.19, 95\% CI 2.80-3.64, $p<0.0001)$.

\section{Late CMV Recurrence}

Late CMV recurrence occurs after day 100 in patients with chronic GVHD and receiving immunosuppressive therapy, but also after cessation of previous prophylaxis or a preemptive strategy. The high-risk group included patients who did not receive an MFD graft, or who developed GVHD despite receiving such a graft, and in addition had more than two episodes of early CMV recurrence and either lymphopenia at day 100 or transplantation from a CMVseronegative donor. The low-risk patients were those without antecedent early recurrence, or those with early recurrence and transplantation for a myeloid malignancy from an MFD without subsequent acute GVHD. Intermediate-risk patients included those who did not fit in either the low-risk group or the high-risk group [64].

From the more than 16,000 allogeneic hematopoietic stem cell transplantations performed in European transplant centers [74], the estimated annual number of patients at risk of CMV recurrence is more than 6000 in the case of $\mathrm{D}+/ \mathrm{R}+\mathrm{CMV}$ serostatus (incidence $39.1 \%)$, more than 3000 in the case of $\mathrm{D}-/$ $\mathrm{R}+\mathrm{CMV}$ serostatus (incidence 20.1\%), more than 4000 in the case of UD/MMD and R+ CMV serostatus (incidence 25.5\%) [61, 74], more than 5000 in the case of acute GVHD of grade 2 or higher (incidence $32.4 \%$ ), and more than 3000 in the case of chronic GVHD (incidence $20.7 \%)[74,75]$.

\section{RISK FACTORS FOR CMV DISEASE IN ALLOGENEIC HEMATOPOIETIC STEM CELL TRANSPLANTATION PATIENTS}

There are three studies with multivariate analysis for risk factors for CMV disease in patients after allogeneic HSCT available in the literature $[18,21,69]$. In all of these studies, $\mathrm{D}-/ \mathrm{R}+$ pretransplant CMV serostatus had a significant role as a risk factor. Two of these studies indicated UD/MMR transplant [18, 67-69] and GVHD $[18,21,69]$ as risk factors. In one study, three other factors were also found: high CMV viral load, female-to-male donor, and year of transplant before 1998 [18]. High CMV viral load was a risk factor not only for development of $\mathrm{CMV}$ disease but also for increased risk of overall death $[18,19,76]$.

Overall, data on CMV disease from 3780 allogeneic hematopoietic stem cell transplantations are available [18, 21, 69], mainly in leukemias; among them the CMV-seropositive rate of recipients was 55.9\% (2114/3780). From the published data, CMV disease occurred in 
Table 2 CMV disease after allogeneic allogeneic hematopoietic stem cell transplantation according to donor/recipient serostatus

\begin{tabular}{lll}
\hline $\begin{array}{l}\text { Recipient CMV } \\
\text { serostatus }\end{array}$ & \multicolumn{2}{l}{ Donor CMV serostatus } \\
\cline { 2 - 3 } & Negative & Positive \\
\hline Negative & $0.2 \%(2 / 813)$ & $3.1 \%(27 / 853)$ \\
Positive & $14.5 \%(187 /$ & $12.9 \%(107 /$ \\
& $1288)$ & $826)$ \\
\hline
\end{tabular}

Raw data from $[18,21,69]$

13.9\% of CMV-seropositive recipients (294/ 2114 ) and in $1.7 \%$ of CMV-seronegative recipients (29/1666) (Table 2). The CMV-seropositive recipients had ninefold higher odds of CMV disease than the CMV-seronegative recipients (OR 9.12, 95\% CI 6.19-13.43, $p<0.0001$ ).

CMV disease occurred in $12.5 \%$ of UD/MMD transplant recipients $(23 / 182)$ and in $4.4 \%$ of MFD transplant recipients $(18 / 412)[63,66,67,69,73]$. The risk of CMV disease is threefold higher in UD/ MMD transplants than in MFD transplants (OR $3.18,95 \%$ CI 1.67-6.06, $p<0.001$ ).

CMV disease occurred in $17.9 \%$ of patients (7/39) with acute GVHD and in $2.4 \%$ of patients (3/123) without acute GVHD [21]. The risk of CMV disease is higher in acute GVHD patients than in those without acute GVHD (OR 8.75, 95\% CI 2.14-35.7, $p<0.001$ ).

Recipient and donor serostatus play a key role also in the development of CMV disease after HSCT. Ljungman et al. [69] found that a patient who was seronegative and who had a seronegative marrow donor developed CMV disease, whereas the corresponding probabilities for seronegative patients with seropositive donors, seropositive patients with seronegative donors, and seropositive patients with seropositive donors were $5.4 \%$, $13.7 \%$, and $11.7 \%$, respectively.

No adverse role of the use of anti-thymocyte globulin or sex mismatch between the donor and the recipient (e.g., female to male) has been proven, whereas the use of sirolimus in GVHD prophylaxis [67] and the use of preemptive prophylaxis had a protective effect against CMV disease, CMV-associated death, and transplantrelated death [69].
With respect to late CMV disease, the rates were comparable in nonmyeloablative (reduced-intensity conditioning) and myeloablative conditioning groups during the first 100 days after HSCT, but reduced-intensity conditioning hematopoietic stem cell transplant recipients had an increased risk of late $\mathrm{CMV}$ disease. The increased risk of late CMV disease was pronounced also in UD/MMD transplants, as well as in patients with acute GVHD, with chronic GVHD, with high CMV DNAemia, and those with early CMV recurrence $[18,64]$.

\section{RISK FACTORS FOR CMV DISEASE IN PATIENTS WITH OTHER HEMATOLOGICAL MALIGNANCIES}

From the reported cases, the cumulative rate of CMV disease after autologous HSCT was 2.05\% (53 of 2576 reported patients) [15, 22-28]. The cumulative rate of CMV disease in patients with hematological malignancies undergoing nontransplant treatment was $1.22 \%$ (77 of 6321 reported patients) $[15,22,25,26,29]$. Both recurrence and disease incidence might obviously be increased when alemtuzumab, rituximab, bortezomib, or fludarabine is used in seropositive patients.

\begin{tabular}{|c|c|c|}
\hline RISK FACTOR & CMV REACTIVATION & CMV DISEASE \\
\hline $\mathrm{D}-/ \mathrm{R}+$ & & \\
\hline GVHD & & \\
\hline UD/MMD & & \\
\hline AGE $(\uparrow)$ & & \\
\hline
\end{tabular}

Fig. 5 Major risk factors for CMV reactivation and CMV disease in allogeneic hematopoietic stem cell transplantation patients according to number of studies indicating statistical significance in multivariate analysis. D- donor negative, GVHD graft-versus-host disease, MMD mismatched donor, $\mathrm{R}+$ recipient positive, UD unrelated donor 


\section{CONCLUSION}

Most studies on risk factors for CMV recurrence or CMV disease after allogeneic HSCT indicate three risk factors: $\mathrm{D}-/ \mathrm{R}+\mathrm{CMV}$ serostatus, acute or chronic GVHD, and UD/MMD transplant (Fig. 5). Increased age is also a risk factor, but it corresponds to the increasing CMV-seropositivity rate in the general population. The three risk factors should be regarded as major risk factors. All other factors should be regarded as minor risk factors, regardless of early or late phase after allogeneic HSCT.

\section{ACKNOWLEDGEMENTS}

The author thanks the editor and anonymous reviewers for their efforts to improve the quality of this article.

Funding. No funding or sponsorship was received for this study or publication of this article.

Authorship. The author meets the International Committee of Medical Journal Editors (ICMJE) criteria for authorship for this manuscript, take responsibility for the integrity of the work as a whole, and has given final approval for the version to be published.

Prior Presentation. The article is based on the lecture "Who is the patient at risk for CMV reactivation?" presented by the author at the symposium "CMV+: what to do with your transplant patient?" sponsored by MSD during the European Congress of Clinical Microbiology and Infectious Diseases in Vienna on April 23, 2017.

Disclosures. Jan Styczynski received an honorarium for giving the lecture "Who is the patient at risk for CMV reactivation?". Jan Styczynski has no other personal, financial, commercial, or academic conflicts of interest with respect to this article.
Compliance with Ethics Guidelines. This article is based on previously conducted studies and does not involve any new studies of human or animal subjects performed by the author.

Open Access. This article is distributed under the terms of the Creative Commons Attribution-NonCommercial 4.0 International License (http://creativecommons.org/licenses/ by-nc/4.0/), which permits any noncommercial use, distribution, and reproduction in any medium, provided you give appropriate credit to the original author(s) and the source, provide a link to the Creative Commons license, and indicate if changes were made.

\section{REFERENCES}

1. Manicklal S, Emery VC, Lazzarotto T, Boppana SB, Gupta RK. The "silent" global burden of congenital cytomegalovirus. Clin Microbiol Rev. 2013;26(1):86-102.

2. Ljungman P, Brandan R. Factors influencing cytomegalovirus seropositivity in stem cell transplant patients and donors. Haematologica. 2007;92(8):1139-42.

3. Ljungman $\mathrm{P}$, de la Camara $\mathrm{R}$, Cordonnier $\mathrm{C}$, et al. Management of CMV, HHV-6, HHV-7 and Kaposisarcoma herpesvirus (HHV-8) infections in patients with hematological malignancies and after SCT. Bone Marrow Transpl. 2008;42(4):227-40.

4. Styczynski J. Managing post-transplant lymphoproliferative disorder. Expert Opin Orphan Drugs. 2017;5(1):19-35.

5. Styczynski J, Reusser P, Einsele H. Management of HSV, VZV and EBV infections in patients with hematological malignancies and after SCT: guidelines from the Second European Conference on Infections in Leukemia. Bone Marrow Transpl. 2009;43(10):757-70.

6. Cannon MJ. Congenital cytomegalovirus (CMV) epidemiology and awareness. J Clin Virol. 2009;46(Suppl 4):S6-10.

7. Hyde TB, Schmid DS, Cannon MJ. Cytomegalovirus seroconversion rates and risk factors: implications for congenital CMV. Rev Med Virol. 2010;20(5):311-26. 
8. Cannon MJ, Schmid DS, Hyde TB. Review of cytomegalovirus seroprevalence and demographic characteristics associated with infection. Rev Med Virol. 2010;20(4):202-13.

9. Ljungman P, Hakki M, Boeckh M. Cytomegalovirus in hematopoietic stem cell transplant recipients. Hematol Oncol Clin North Am. 2011;25(1):151-69.

10. Reusser P, Riddell SR, Meyers JD, Greenberg PD. Cytotoxic T-lymphocyte response to cytomegalovirus after human allogeneic bone marrow transplantation: pattern of recovery and correlation with cytomegalovirus infection and disease. Blood. 1991;78(5):1373-80.

11. Boeckh M, Leisenring W, Riddell SR, et al. Late cytomegalovirus disease and mortality in recipients of allogeneic hematopoietic stem cell transplants: importance of viral load and T-cell immunity. Blood. 2003;101(2):407-14.

12. Ljungman $\mathrm{P}$, Boeckh $\mathrm{M}$, Hirsch $\mathrm{HH}$, et al. Definitions of cytomegalovirus infection and disease in transplant patients for use in clinical trials. Clin Infect Dis. 2017;64(1):87-91.

13. Arav-Boger R. Strain variation and disease severity in congenital cytomegalovirus infection. in search of a viral marker. Infect Dis Clin North Am. 2015;29(3):401-14.

14. Renzette N, Pokalyuk C, Gibson L, et al. Limits and patterns of cytomegalovirus genomic diversity in humans. Proc Natl Acad Sci USA. 2015;112(30):E4120-8.

15. Styczynski J, Czyzewski K, Wysocki M, et al. Increased risk of infections and infection-related mortality in children undergoing haematopoietic stem cell transplantation compared to conventional anticancer therapy: a multicentre nationwide study. Clin Microbiol Infect. 2016;22(2):179.e1-10.

16. Schmidt-Hieber M, Labopin M, Beelen D, et al. CMV serostatus still has an important prognostic impact in de novo acute leukemia patients after allogeneic stem cell transplantation: a report from the Acute Leukemia Working Party of EBMT. Blood. 2013;122(19):3359-64.

17. Teira P, Battiwalla M, Ramanathan M, et al. Early cytomegalovirus reactivation remains associated with increased transplant-related mortality in the current era: a CIBMTR analysis. Blood. 2016;127(20):2427-38.

18. Nakamae H, Kirby KA, Sandmaier BM, et al. Effect of conditioning regimen intensity on CMV infection in allogeneic hematopoietic cell transplantation. Biol Blood Marrow Transpl. 2009;15(6):694-703.
19. Green ML, Leisenring W, Xie H, et al. Cytomegalovirus viral load and mortality after haemopoietic stem cell transplantation in the era of pre-emptive therapy: a retrospective cohort study. Lancet Haematol. 2016;3(3):e119-27.

20. Schuster MG, Cleveland AA, Dubberke ER, et al. Infections in hematopoietic cell transplant recipients: results from the organ transplant infection project, a multicenter, prospective, cohort study. Open Forum Infect Dis. 2017;4(2):ofx050.

21. Ljungman P, Perez-Bercoff L, Jonsson J, et al. Risk factors for the development of cytomegalovirus disease after allogeneic stem cell transplantation. Haematologica. 2006;91(1):78-83.

22. Han XY. Epidemiologic analysis of reactivated cytomegalovirus antigenemia in patients with cancer. J Clin Microbiol. 2007;45(4):1126-32.

23. Inazawa $\mathrm{N}$, Hori $\mathrm{T}$, Nojima $\mathrm{M}$, et al. Virus reactivations after autologous hematopoietic stem cell transplantation detected by multiplex PCR assay. J Med Virol. 2017;89(2):358-62.

24. Jain T, John J, Kotecha A, et al. Cytomegalovirus infection in autologous stem cell transplant recipients in the era of rituximab. Ann Hematol. 2016;95(8):1323-7.

25. Marchesi F, Pimpinelli F, Ensoli F, Mengarelli A. Cytomegalovirus infection in hematologic malignancy settings other than the allogeneic transplant. Hematol Oncol. 2017. https://doi.org/10.1002/hon. 2453

26. Piukovics K, Terhes G, Gurbity-Palfi T, et al. Cytomegalovirus infection in patients with haematological diseases and after autologous stem cell transplantation as consolidation: a single-centre study. Ann Hematol. 2017;96(1):125-31.

27. Mengarelli A, Annibali O, Pimpinelli F, et al. Prospective surveillance vs clinically driven approach for CMV reactivation after autologous stem cell transplant. J Infect. 2016;72(2):265-8.

28. Marchesi F, Pimpinelli F, Gumenyuk S, et al. Cytomegalovirus reactivation after autologous stem cell transplantation in myeloma and lymphoma patients: a single-center study. World J Transpl. 2015;5(3):129-36.

29. Jain R, Trehan A, Mishra B, Singh R, Saud B, Bansal D. Cytomegalovirus disease in children with acute lymphoblastic leukemia. Pediatr Hematol Oncol. 2016;33(4):239-47.

30. Skoetz N, Bauer K, Elter T et al. Alemtuzumab for patients with chronic lymphocytic leukaemia. Cochrane Database Syst Rev. 2012;(2):CD008078. 
31. Hilal T, Slone S, Peterson S, Bodine C, Gul Z. Cytomegalovirus reactivation is associated with a lower rate of early relapse in myeloid malignancies independent of in vivo T cell depletion strategy. Leuk Res. 2017;57:37-44.

32. Ramanan P, Razonable RR. Cytomegalovirus infections in solid organ transplantation: a review. Infect Chemother. 2013;45(3):260-71.

33. Natori Y, Humar A, Husain S, et al. Recurrence of CMV infection and the effect of prolonged antivirals in organ transplant recipients. Transplantation. 2017;101(6):1449-54.

34. Nagai S, Mangus RS, Anderson E, et al. Cytomegalovirus infection after intestinal/multivisceral transplantation: a single-center experience with 210 cases. Transplantation. 2016;100(2):451-60.

35. Selvey LA, Lim WH, Boan P, et al. Cytomegalovirus viraemia and mortality in renal transplant recipients in the era of antiviral prophylaxis. Lessons from the western Australian experience. BMC Infect Dis. 2017;17(1):501.

36. Kamar N, Mengelle C, Esposito L, et al. Predictive factors for cytomegalovirus reactivation in cytomegalovirus-seropositive kidney-transplant patients. J Med Virol. 2008;80(6):1012-7.

37. Cipe FE, Dogu F, Aytekin C, et al. HLA-haploidentical transplantations for primary immunodeficiencies: a single-center experience. Pediatr Transpl. 2012;16(5):451-7.

38. Al-Herz W, Zainal ME, Alenezi HM, Husain K, Alshemmari SH. Performance status and deaths among children registered in Kuwait National Primary ImmunoDeficiency Disorders Registry. Asian Pac J Allergy Immunol. 2010;28(2-3):141-6.

39. Al-Herz W, Moussa MA. Survival and predictors of death among primary immunodeficient patients: a registry-based study. J Clin Immunol. 2012;32(3):467-73.

40. Durier N, Ananworanich J, Apornpong T, et al. Cytomegalovirus viremia in Thai HIV-infected patients on antiretroviral therapy: prevalence and associated mortality. Clin Infect Dis. 2013;57(1):147-55.

41. Brantsaeter $A B$, Holberg-Petersen $M$, Jeansson $S$, Goplen AK, Bruun JN. CMV quantitative PCR in the diagnosis of CMV disease in patients with HIV-infection-a retrospective autopsy based study. BMC Infect Dis. 2007;7:127.

42. Reitter A, Buxmann H, Haberl AE, et al. Incidence of CMV co-infection in HIV-positive women and their neonates in a tertiary referral centre: a cohort study. Med Microbiol Immunol. 2016;205(1):63-71.

43. Fielding K, Koba A, Grant AD, et al. Cytomegalovirus viremia as a risk factor for mortality prior to antiretroviral therapy among HIV-infected gold miners in South Africa. PLoS One. 2011;6(10):e25571.

44. Luo B, Sun J, Cai R, et al. Spectrum of opportunistic infections and risk factors for in-hospital mortality of admitted AIDS patients in Shanghai. Medicine (Baltimore). 2016;95(21):e3802.

45. Xiao J, Gao G, Li Y, et al. Spectrums of opportunistic infections and malignancies in HIV-infected patients in tertiary care hospital, China. PLoS One. 2013;8(10):e75915.

46. Varo R, Buck WC, Kazembe PN, Phiri S, Andrianarimanana $\mathrm{D}$, Weigel $\mathrm{R}$. Seroprevalence of CMV, HSV-2 and HBV among HIV-infected malawian children: a cross-sectional survey. J Trop Pediatr. 2016;62(3):220-6.

47. Ford N, Shubber Z, Saranchuk P, et al. Burden of HIV-related cytomegalovirus retinitis in resourcelimited settings: a systematic review. Clin Infect Dis. 2013;57(9):1351-61.

48. Chiotan C, Radu L, Serban R, Cornacel C, Cioboata $\mathrm{M}$, Anghel A. Cytomegalovirus retinitis in HIV/ AIDS patients. J Med Life. 2014;7(2):237-40.

49. Sepehrvand N, Khameneh ZR, Eslamloo HR. Survey the seroprevalence of CMV among hemodialysis patients in Urmia, Iran. Saudi J Kidney Dis Transpl. 2010;21(2):363-7.

50. Vilibic-Cavlek T, Kolaric B, Beader N, Vrtar I, Tabain I, Mlinaric-Galinovic G. Seroepidemiology of cytomegalovirus infections in Croatia. Wien Klin Wochenschr. 2017;129(3-4):129-35.

51. Pliquett RU, Klein C, Grunewald T, Ruf BR, Beige J. Lack of evidence for systemic cytomegalovirus reactivation in maintenance hemodialysis patients. Eur J Clin Microbiol Infect Dis. 2011;30(12):1557-60.

52. Wang S, Wang T, Zhang W, et al. Cohort study on maternal cytomegalovirus seroprevalence and prevalence and clinical manifestations of congenital infection in China. Medicine (Baltimore). 2017;96(5):e6007.

53. Bialas KM, Swamy GK, Permar SR. Perinatal cytomegalovirus and varicella zoster virus infections: epidemiology, prevention, and treatment. Clin Perinatol. 2015;42(1):61-75. 
54. Kelly MS, Benjamin DK, Puopolo KM, et al. Postnatal cytomegalovirus infection and the risk for bronchopulmonary dysplasia. JAMA Pediatr. 2015;169(12):e153785.

55. Kenneson A, Cannon MJ. Review and meta-analysis of the epidemiology of congenital cytomegalovirus (CMV) infection. Rev Med Virol. 2007;17(4):253-76.

56. Dollard SC, Grosse SD, Ross DS. New estimates of the prevalence of neurological and sensory sequelae and mortality associated with congenital cytomegalovirus infection. Rev Med Virol. 2007;17(5):355-63.

57. Karimian P, Yaghini O, Nasr Azadani $\mathrm{H}$, et al. Prevalence, characteristics, and one-year follow-up of congenital cytomegalovirus infection in Isfahan City, Iran. Interdiscip Perspect Infect Dis. 2016;2016:7812106.

58. Pokorska-Spiewak M, Niezgoda A, Golkowska M, et al. Recommendations for the diagnosis and treatment of CMV infections. Polish Society of Epidemiology and Infectious Diseases. Przegl Epidemiol. 2016;70(2):297-310.

59. Ogonek J, Kralj Juric M, Ghimire S, et al. Immune reconstitution after allogeneic hematopoietic stem cell transplantation. Front Immunol. 2016;7:507.

60. Ganepola S, Gentilini C, Hilbers U, et al. Patients at high risk for CMV infection and disease show delayed CD8+ T-cell immune recovery after allogeneic stem cell transplantation. Bone Marrow Transpl. 2007;39(5):293-9.

61. Ljungman P, Brand R, Hoek J, et al. Donor cytomegalovirus status influences the outcome of allogeneic stem cell transplant: a study by the European Group for Blood and Marrow Transplantation. Clin Infect Dis. 2014;59(4):473-81.

62. Zhou W, Longmate J, Lacey SF, et al. Impact of donor CMV status on viral infection and reconstitution of multifunction CMV-specific $\mathrm{T}$ cells in CMV-positive transplant recipients. Blood. 2009;113(25):6465-76.

63. Takenaka K, Nishida T, Asano-Mori Y, et al. Cytomegalovirus reactivation after allogeneic hematopoietic stem cell transplantation is associated with a reduced risk of relapse in patients with acute myeloid leukemia who survived to day 100 after transplantation: the Japan Society for Hematopoietic Cell Transplantation Transplantation-related Complication Working Group. Biol Blood Marrow Transpl. 2015;21(11):2008-16.

64. Ozdemir E, Saliba RM, Champlin RE, et al. Risk factors associated with late cytomegalovirus reactivation after allogeneic stem cell transplantation for hematological malignancies. Bone Marrow Transpl. 2007;40(2):125-36.

65. Nichols WG, Corey L, Gooley T, Davis C, Boeckh M. High risk of death due to bacterial and fungal infection among cytomegalovirus (CMV)seronegative recipients of stem cell transplants from seropositive donors: evidence for indirect effects of primary CMV infection. J Infect Dis. $2002 ; 185(3): 273-82$

66. Walker CM, van Burik JA, De For TE, Weisdorf DJ. Cytomegalovirus infection after allogeneic transplantation: comparison of cord blood with peripheral blood and marrow graft sources. Biol Blood Marrow Transpl. 2007;13(9):1106-15.

67. Marty FM, Bryar J, Browne SK, et al. Sirolimus-based graft-versus-host disease prophylaxis protects against cytomegalovirus reactivation after allogeneic hematopoietic stem cell transplantation: a cohort analysis. Blood. 2007;110(2):490-500.

68. Nichols WG, Corey L, Gooley T, et al. Rising pp65 antigenemia during preemptive anticytomegalovirus therapy after allogeneic hematopoietic stem cell transplantation: risk factors, correlation with DNA load, and outcomes. Blood. 2001;97(4):867-74.

69. Ljungman P, Aschan J, Lewensohn-Fuchs I, et al. Results of different strategies for reducing cytomegalovirus-associated mortality in allogeneic stem cell transplant recipients. Transplantation. 1998;66(10):1330-4.

70. Mise J, Dembitz V, Banfic H, Visnjic D. Combined inhibition of PI3K and mTOR exerts synergistic antiproliferative effect, but diminishes differentiative properties of rapamycin in acute myeloid leukemia cells. Pathol Oncol Res. 2011;17(3):645-56.

71. Kudchodkar SB, Yu Y, Maguire TG, Alwine JC. Human cytomegalovirus infection alters the substrate specificities and rapamycin sensitivities of raptor- and rictor-containing complexes. Proc Natl Acad Sci U S A. 2006;103(38):14182-7.

72. Reddehase MJ, Balthesen M, Rapp M, Jonjic S, Pavic I, Koszinowski UH. The conditions of primary infection define the load of latent viral genome in organs and the risk of recurrent cytomegalovirus disease. J Exp Med. 1994;179(1):185-93.

73. Lin TS, Zahrieh D, Weller E, Alyea EP, Antin JH, Soiffer RJ. Risk factors for cytomegalovirus reactivation after CD6+ T-cell-depleted allogeneic bone marrow transplantation. Transplantation. 2002;74(1):49-54. 
74. Passweg JR, Baldomero H, Bader P, et al. Impact of drug development on the use of stem cell transplantation: a report by the European Society for Blood and Marrow Transplantation (EBMT). Bone Marrow Transpl. 2017;52(2):191-6.

75. Styczynski J, Tridello G, Gil L, et al. Impact of donor Epstein-Barr virus serostatus on the incidence of graft-versus-host disease in patients with acute leukemia after hematopoietic stem-cell transplantation: a study from the Acute Leukemia and
Infectious Diseases Working Parties of the European Society for Blood and Marrow Transplantation. J Clin Oncol. 2016;34(19):2212-20.

76. Emery VC, Sabin CA, Cope AV, Gor D, HassanWalker AF, Griffiths PD. Application of viral-load kinetics to identify patients who develop cytomegalovirus disease after transplantation. Lancet. 2000;355(9220):2032-6. 